\title{
MICROFILARIASIS OF THE MALE BREAST
}

\author{
Goonj Johri ${ }^{1}$, Ankur Sharma², Mayank Mathur³, Osman Musa ${ }^{4}$
}

${ }^{1}$ Assistant Professor, Department of General Surgery, Era's Lucknow Medical College, Lucknow.

2 Assistant Professor, Department of General Surgery, IIMS\&R, Integral University, Lucknow.

${ }^{3}$ Senior Resident, Department of General Surgery, Era's Lucknow Medical College, Lucknow.

4 Professor and Head, Department of General Surgery, Era's Lucknow Medical College, Lucknow.

\section{ABSTRACT}

Filariasis is a major public health problem in the tropics. The involvement of breast, more so in men, is a rare event even in the endemic areas. In this case report, we present an elderly man with a painless breast lump with microfilarial involvement. A triple evaluation was done initially to rule out malignancy. Examination revealed a discrete, firm, retroareolar lump. Ultrasonography showed soft tissue thickening and edema in subcutaneous plane suggestive of inflammatory pathology. FNAC revealed microfilariae in a background of leucocytes. Diagnosis of Bancroftian filariasis was made and patient was started on oral diethylcarbamazine. After 3 weeks of treatment the swelling resolved completely and the patient is asymptomatic for more than one year.

\section{KEYWORDS}

Male Breast Lump, Microfilaria, FNAC.

HOW TO CITE THIS ARTICLE: Goonj Johri, Ankur Sharma, Mayank Mathur, Osman Musa. "Microfilariasis of the Male Breast." Journal of Evolution of Medical and Dental Sciences 2015; Vol. 4, Issue 101, December 17; Page: 16705-16706,

DOI: $10.14260 /$ jemds/2015/2498

\section{INTRODUCTION}

Filariasis is an infectious parasitic disease and is regarded as a major public health problem in the tropical countries of Africa, Southern America and Asia.(1) In India it is endemic in Orissa, Uttar Pradesh, Bihar, Andhra Pradesh, Tamil Nadu and Gujarat. The nematode Wuchereria bancrofti accounts for majority of cases worldover followed by Brugia malayi and Brugia timori.(2) infected mosquitoes serve as vectors and humans are the definitive host.

The commonest presentation is lymphatic filariasis which usually involves lower extremities, external genitalia, retroperitoneal tissue and axilla causing lymphedema, elephantiasis, hydrocele and Ram horn penis. Involvement of the breast is rare, more so in the males.(2)

\section{CASE REPORT}

A 50-year-old male, resident of Uttar Pradesh presented with a gradually progressive painless lump in right breast of 2 months' duration. There was no history of fever, cough, weight loss or trauma. No significant treatment or drug history or chronic alcoholism. There was no history of breast cancer in the family. On examination the lump was $3 \times 2 \mathrm{~cm}$ in size, retroareolar, firm in consistency and freely mobile. Overlying skin was free. There was no axillary lymphadenopathy. (Figure 1). Opposite breast was normal. Systemic examination was normal. A provisional diagnosis of breast cancer was made and further evaluation was carried out. A High resolution ultrasonography done with a $7.5 \mathrm{MHz}$ transducer showed mild soft tissue thickening and edema in subcutaneous plane in retroareolar region suggestive of inflammatory etiology.

Financial or Other, Competing Interest: None

Submission 25-11-2015, Peer Review 26-11-2015,

Acceptance 10-12-2015, Published 17-12-2015.

Corresponding Author:

Dr. Goonj Johri,

Assistant Professor,

Department of General Surgery,

ELMC, Sarfarazganj,

Hardoi Road,

Lucknow-226003.

E-mail:goonjjohri@gmail.com

DOI:10.14260/jemds/2015/2498
For further evaluation, Fine Needle Aspiration Cytology (FNAC) was done with 23-gauge needle, which showed microfilariae and clusters of epithelial cells with pleomorphic nuclei (fine chromatin) and eosinophillic cytoplasm in a background of leucocytes. (Figure 2). There was no evidence of microfilaria on peripheral blood smear. The patient was given a single dose of oral albendazole $400 \mathrm{mg}$ and started on 3 weeks therapy with oral Diethylcarbamazine $100 \mathrm{mg}$ q 8 hourly. The patient presented for first followup after 3 weeks, at which the swelling had completely resolved. Patient has remained asymptomatic for more than one year now.

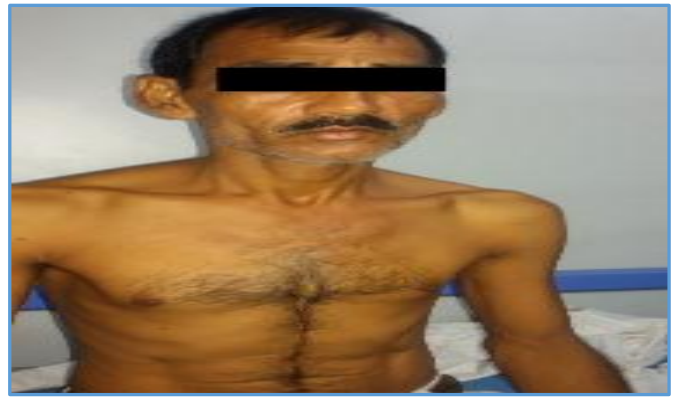

Fig. 1: Clinical photograph showing Right breast larger than the Left (Gynaecomastia) due to retroareolar lump

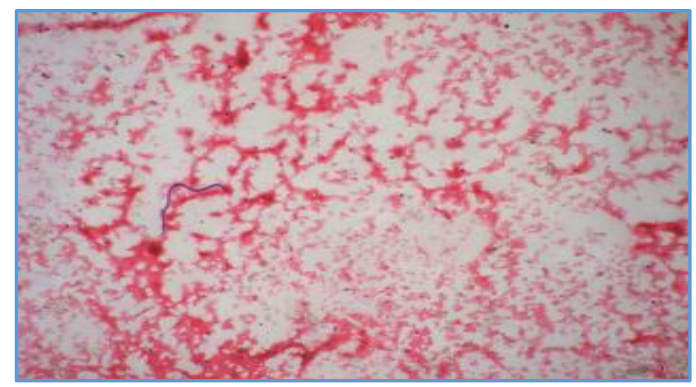

Fig. 2: Photomicrograph of Fine needle aspiration cytology (FNAC) smear showing clusters of epithelial cells and Microfilaria. These cells have mildly pleomorphic nucleus (Fine chromatin) and eosinophilic cytoplasm. Background shows haemorrhage and blood leucocytes (H\&E 10X). H\&E = Haematoxylin and Eosin stain 


\section{DISCUSSION}

Filariasis is a parasitic disease caused by infection with a roundworm of Filarioideae superfamily. Humans are definitive hosts in eight known filarial nematodes. The disease manifests as two distinct clinical types- Lymphatic filariasis caused by parasite in lymphatic system and occult filariaisis caused by immune hyper responsiveness of the human host or tropical pulmonary eosinophilia.(2)

Filariasis is a global health problem. More than 1.4 billion people live in endemic areas of these 120 million are infected and 40 million have overt disease.(3) Lymphatic filariasis is a major health problem in India with most infections caused by Wuchereria bancrofti (95\%), Brugia malai and Brugia timori. Heavily infected areas are Uttar Pradesh, Bihar, Jharkhand, Andhra Pradesh, Orrisa, Tamil Nadu, Kerala and Gujarat.(3)

The adult worms which usually stay in lymphatic tissue and release early larval forms in bloodstream known as microfilariae into host bloodstream. Circulating microfilariae can be picked up with a blood meal by the arthropod vector and transmitted to a new host.(4)

Depending on whether microfilaria can be detected in the peripheral blood, patients are described as microfilaraemic or amicrofilareamic. Man is a natural host. All ages are susceptible. In endemic areas the microfilaria rate is higher in men. Culex, Anopheles and Aedes species of mosquito are vectors for W. Bancrofti. (3)

The disease may be asymptomatic or there can be acute episodes of local inflammation involving skin, lymph nodes and lymphatic vessels. Chronic disease in endemic communities can manifest in men in the form of genital damage, especially hydrocele, funiculitis, epididymitis and elephantiasis of the penis and scrotum. In women, the vulva or breast may be involved. An entire arm or leg maybe affected in both.(5) Involvement of the male breast is extremely rare and has not been reported in literature.

Filariasis of female breast is occasionally seen in tropical areas where filariasis is endemic. The larvae enter the lymphatic vessels causing lymphangitis, fibrosis and disruption of lymphatic drainage and presents as a unilateral painless solitary non-tender breast mass, commonly in the upper and outer quadrant.(5) Sometimes, skin over the nodule may be hyperemic with changes of peau d'orange and the axillary nodes may be enlarged mimicking inflammatory carcinoma breast.(6)

Microfilaria of W. bancrofti circulate in the peripheral blood with regular nocturnal periodicity and maximum density had been reported between $10 \mathrm{pm}$ and 2 am.(4) Tests used for diagnosis include demonstration of microfilariae in the peripheral blood or skin and detection of filarial antigens and antibody.(5) For detection of microfilaria thick and thin blood smears are made and stained with Haematoxylin or Giemsa stains.
Ultrasound is a valuable tool in the diagnosis of cases of lymphatic filariasis. Amaral et al.(7) had first reported the use of ultrasound to visualize adult worms of W. bancrofti in the scrotal area of infected men. They described a continuous, distinctive and specific pattern of worm movement called the "Filarial dance" sign.(5)

Microfilariae may sometimes be detected in fine needle aspirates from the breast lump or lymph node, even though thick peripheral blood smear examination may not reveal them similar to our case.(2) These worms can later calcify and these calcifications are well visualized on breast mammograms.(5) FNAC shows Microfilariae and clusters of epithelial cells in a background of leucocytes. In one case study the cellular smears from the aspirate showed gravid adult filarial worms with a preserved outer cuticle layer. Numerous microfilariae were seen coming out from the paired uteri.(1)

Filarial antigen may be detected in patient serum by EIA using monoclonal antibodies. Polymerase chain reactionbased immunological blood test for Wuchereria bancrofti can also be a helpful adjunct the diagnostic work-up.(4)

Diethylcarbamazine is safe and effective causing rapid disappearance of microfilaria from circulation. The effect on adult worm is uncertain. The recommended dose is $6 \mathrm{mg} / \mathrm{kg}$ body weight per day in divided doses given orally for 12 days. It may be combined with either Albendazole or Ivermectin for mass therapy in endemic areas.(3) Vectors can be controlled by Anti larval measures and minor environmental measures.

\section{CONCLUSION}

This case report underlines the fact that filariasis should be considered as one of the differential diagnosis of breast lumps in men, especially in endemic areas.

\section{REFERENCES}

1. Chakrabarti I, Das V, Halder B, et al. Adult filarial worm in the aspirate from a breast lump mimicking fibroadenosis. Trop Parasitol 2011;1:129-31.

2. Bhattacharjee PK, Ray RP, Halder S. Filariasis of breast: An unusual presentation. Ann Trop Med Public Health 2012;5:376-8.

3. Park K. Park's Textbook of Preventive and Social Medicine. 23rd edition. Jabalpur: Bhanot; 2015.

4. Paniker Jayaram CK, Ghosh Sougata. Paniker's Textbook of Medical Parasitology. 7th ed. New Delhi: Jaypee Brothers Medical Publishers (P) Ltd; 2013.

5. Upadhyaya V, Upadhyaya DN, Sarkar S. An interesting case of breast filariasis. Indian J Radiol Imaging 2006;16:915-7.

6. Lahiri VL. Microfilariae in nipple secretion. Acta Cytol 1975;19:154-5.

7. Amaral F, Dreyer G, Figueredo-Silva J, et al. Adult worms detected by ultrasonography in human bancroftian filariasis. Am J Trop Med Hyg 1994;50:753-7. 